\title{
Construcción de la protesta feminista en hashtags: aproximaciones desde el análisis de redes sociales*
}

\author{
Building the feminist's protest in hashtags: approaching from Social Network \\ Analysis
}

\section{Daniela Esquivel Domínguez}

Universidad Nacional Autónoma de México, Ciudad de México, México dan.esquivel23agmail.com

\section{Resumen}

El presente artículo establece pautas para la identificación del desarrollo y el vínculo de las protestas feministas internacionalmente, que se enfocan en visibilizar diversos tipos de violencia contra las mujeres, narrados desde la experiencia a través del uso del hashtag en la plataforma Twitter. Por medio de la documentación de los hashtags que alcanzaron Trending Topic a nivel nacional e internacional y su impacto en la cotidianeidad social entre el 2009 y 2019, se elaboró una red a partir de un análisis de co-palabras y la estructura se definió a partir del algoritmo Kamada Kawai. El resultado de esta red posibilita identificar tres grupos temáticos sobre los cuales se define la agenda feminista en redes sociodigitales: la violencia sexual, los derechos reproductivos y los derechos laborales de las mujeres. El mapeo de estos hashtags ha permitido visualizar una estructura cognitiva articulada.

Palabras clave: análisis de redes sociales, análisis de co-palabras, violencia contra las mujeres, análisis feminista, experiencia

\begin{abstract}
This paper establishes guidelines to identify the development of international feminist's protests, oriented towards risins awareness about different types of violence against women, tolded through the experience, via hashtags on Twitter. The documentation of the hashtags that turnedinto national and international Trending Topic, and its impact on social daily life between 2009 and 2019, were used to build up a network using co-words analysis and the structure was define by Kamada Kawai. This network allows to identify three main topics of the feminist agenda on social networks: sexual violence, reproductive rights and women's labor rights. This mapping acknowledges an articulated cognitive structure.
\end{abstract}

Key words: social network analysis, co-word analysis, violence against women, feminist analysis, experience

* Este artículo forma parte del trabajo de investigación del Seminario Taller de Redes UNAM 


\section{Introducción}

La importancia de analizar la forma de organización de las protestas feministas lleva directamente a repensar la configuración del feminismo como movimiento social y académico a lo largo de la historia y que ha devenido en procesos coyunturales cuyas implicaciones han sido políticas, sociales, culturales y económicas (Lagarde, 1996; Valcárcel, 2001; De las Heras, 2008; Castañeda, 2008). Ante la emergencia de un conjunto de movimientos organizados desde los hashtags que denuncian la violencia contra las mujeres del sistema patriarcal, surge la necesidad de historizar y analizar la forma en la que estas protestas se conjugan entre la dimensión online y offline.

Es por eso que, entre sus objetivos, los feminismos parten de la búsqueda de develar y desmontar las relaciones de poder genéricas tanto en el ámbito privado -aquel que se refiere al espacio doméstico y de la reproducción- como del público -el de la política, la ciencia y la académica- (Fraser, 1990; Cobo, 2008; De Miguel \& Boix, 2002) y, por lo tanto, resignificar la participación de las mujeres en ambos. De ahí que sea fundamental analizar cómo, en las actuales dinámicas sociales, internet y las redes sociodigitales, funcionan como espacios públicos para la enunciación con la capacidad de facilitar la discusión política (Papacharissi, 2002), a partir de recuperar la agencia de los y las usuarias (os), y comprender "el potencial de la tecnología dentro del contexto social, político, cultural y económico que le da vida" (Papacharissi, 2009: 230-231)

A partir de esta observación, es posible denotar que una de las características claramente definidas en las protestas feministas en internet y redes sociodigitales es la constante enunciación y visibilización de las experiencias de violencia que las mujeres sufren en su cotidianidad. En el caso de Twitter, estas experiencias son discursivamente articuladas por medio de hashtags, cuya relevancia y circulación es definido por las usuarias, lo que lleva a reafirmar su agencia para reivindicar su participación política.

El abordaje de estas dinámicas por medio del análisis de redes sociales (ARS), parte de una perspectiva que retoma esta agencia y "considera a las conexiones entre entidades y a los patrones, an- tecedentes y consecuencia de las mismas" (Faust, 2002, p.1). Esta perspectiva analiza cómo interactúan y se vinculan entre ellas las diversas protestas feministas $y$, por lo tanto, observa un "conjunto de nodos socialmente relevantes conectado con una o más relaciones" (Marin \& Wellman, 2011, p.12).

Estos elementos se proponen repensar el quehacer de las mujeres en el espacio público de Internet y en redes sociodigitales, como acciones que reivindican la participación política a partir de la narración de experiencias; y, en conjunto con el ARS (que permiten visualizar cómo se vinculan entre ellos), los aportes de esta investigación consideran que esta serie de protestas que se originan desde principios del 2009 en la plataforma Twitter, no son discursos espontáneos sin origen definido. Más bien, son discursos cuya guía y lógica se encuentran a la base del movimiento feminista histórico y son resignificados en estos espacios a partir de la agencia propia de las usuarias y, por lo tanto, conforman una estructura cognitiva articulada y consistente.

En conjunto, el ejercicio y análisis de esta red establece pautas para documentar y mapear cómo hay una continua construcción de los movimientos feministas a nivel nacional e internacional en estos espacios y que, partiendo de su potencialidad, se permean como herramientas para la organización estratégica y política de las mujeres.

\section{Marco Teórico}

Samara de las Heras refiere que desde el siglo XIX, "el feminismo aparece, por primera vez, como un movimiento social de carácter internacional, con una identidad autónoma teórica y organizativa que ocupa un importante lugar en el seno de los otros grandes movimientos sociales: el socialismo y el anarquismo" (De las Heras, 2008, p. 53). A pesar del extendido cuestionamiento sobre la participación tanto social y política de las mujeres, es el lema "lo personal es político" como eje de reflexión teórica a finales de los sesenta (De las Heras, 2008, p. 56), lo que llevó a diversas feministas a re-pensar y re-elaborar desde este mismo paradigma: ¿cómo hacer ciencia desde las mujeres? A partir de la década de los ochenta, autoras como Sandra Harding (1998), Eli Bartra (1998), Donna Haraway (1991), 
Teresa De Lauretis (1987), entre otras, definirían la posibilidad de tener una metodología y teoría feminista y cuáles serían sus implicaciones.

Mientras que Sandra Harding refiere "que las teorías tradicionales han sido aplicadas de manera tal que hacen difícil comprender la participación de las mujeres en la vida social [...]" (Harding, 1998, p.13), Haraway reflexiona en torno a la marcada visión androcéntrica, donde "los esquemas que limitan el conocimiento son teorizados como actitudes de poder y no como actitudes que buscan la verdad" (Haraway, 1991, p.315). Ambas autoras insisten en la necesidad de repensar, no únicamente la objetividad en la ciencia, sino en cómo la objetividad científica androcéntrica piensa y considera a las mujeres y a las minorías poblacionales que giran en torno a la periferia discursiva hegemónica.

Desde este punto, la investigación feminista se propone hacer a las mujeres el centro de la investigación, con “la convicción de retomar la experiencia de las mujeres como recurso empírico y teórico" (Castañeda, 2008, p. 83) y donde esta implica "no solamente un punto de vista acerca de qué es el conocimiento, sino que tiene connotaciones políticas y éticas que diluyen los limites disciplinarios tradicionales" (Fernández, 2012, p. 63) que como eje de análisis, permite recuperar historias y narrativas de la cotidianidad, en las cuales se incorporan los discursos de dominación, pero también de resistencia (Stone-Mediatore, 1999; Mohanty, 2003; Fernández, 2012). Por esta razón, Chandara Mohanty considera que la experiencia debe abordarse desde el lenguaje como base de la producción de conocimiento de las sujetas, y situarla en "un análisis económico-político global" (StoneMediatore, 1999). Asimismo, implica historizar sus narrativas y reconocer su capacidad de agencia (Mohanty, 2003), porque es donde se construyen y se repiensan las diversas formas de dominación que están presentes, tanto en el lenguaje como en el cuerpo (Stone-Mediatore, 1999).

Desde este razonamiento, la experiencia no debe ser tratada como "la verdad", más bien, como un conocimiento situado dentro de determinados momentos históricos capaz de "contribuir a una conciencia de la comunidad que sustituye la oposición entre la vida pública y la privada" (Stone-Mediatore, 1999) para matizar la realidad y crear significados más allá de la visión binaria, mecánica y hegemónica (Haraway, 1991). A pesar que Mohanty es enfática sobre evitar la generación de discursos que esencialicen 'las experiencias de las mujeres' (Mohanty, 2003), es importante reflexionar sobre las experiencias comunes que viven, específicamente las que respectan a la violencia sobre el cuerpo y la sexualidad.

\subsection{Redes sociodigitales: espacio y esfera pública}

Partiendo de la experiencia como categoría de análisis, el presente artículo propone comprender cómo Twitter se ha conformado como un espacio para colocar la experiencia como punto de reflexión para matizar los discursos hegemónicos. De esta manera, uno de los elementos que hay que considerar para realizar este análisis implica tomar en cuenta el papel que han jugado particularmente las redes sociodigitales ${ }^{2}$ y cómo estas, "proveen información y herramientas que pueden extender el rol del público en la arena social y política" ${ }^{3}$ (Papacharissi, 2002:10).

Hay que partir de situar el contexto en el cual las plataformas son "moldeadas y formadas por una ecología cívica" (Papacharissi, 2011, p.10), donde las usuarias fungen como agentes (Papacharissi, 2009) y a las plataformas como herramientas que forman parte de diversos escenarios, donde confluyen intereses tanto políticos como económicos. Por esta razón es importante comprender que tanto la tecnología como los espacios mediados por ella no son neutrales, pues responden a estructuras de poder (Van Dijk, 2006; Papacharissi, 2009; Kranzberg, 1985).

El impacto que han tenido las redes sociodigitales en este contexto es que se conforman como centros de encuentro para el debate público (Castells, 2009,2010 ) y de discusión política (Toret, 2013), lo que ha permitido construirlas como espacios públicos (Papacharissi 2002, 2009; Khan et. al 2012) en tanto pueden configurar un territorio para "expresar y publicar opiniones sobre asuntos del interés común" (Habermas et al, 1964). Papacharissi propone en esta línea argumentativa que internet cumple con una serie de elementos para congregar a diferentes sectores y públicos en un foro de deliberación política (Papacharissi, 2002, p.11). 
Pero defiende que, en ausencia de un acceso garantizado en igualdad de condiciones a diferentes sectores de la sociedad, así como la estructura privada y comercial de internet, este puede proveer "un espacio público, pero no constituye una esfera pública" (Papacharissi, 2002, p.13). Esta reflexión se fundamenta en el concepto de esfera pública de Habermas, como "el ámbito de la vida social donde la opinión pública puede ser formada" (Habermas et al, 1964), quién también supone que, se garantiza el acceso a todos los ciudadanos para conversar sobre algo de interés común y conformarse como un público (Habermas et al, 1964).

Aunque prevalezcan brechas de acceso y uso ${ }^{4}$ entorno a internet y a las redes sociodigitales, hay que poner en perspectiva la diversidad de dinámicas que se construyen entre las dimensiones online y offline en la actualidad ${ }^{5}$. En este sentido, Thompson (2011) propone ser reflexivos ante la concepción clásica de la esfera pública para percibir otras posibilidades. En este contexto las dinámicas que presuponen las redes sociodigitales no se dan en un vacío. Es decir, estas se definen dentro de un mundo con procesos que ya están mediatizados a través de las lógicas planteadas por los medios masivos de comunicación (Raimondo et. al, 2016). Arguyendo a Thompson, la esfera pública es un espacio de aparición, un proceso de volverse visible y recrear realidades a la vista de otros (Thompson, 2011, p.22).

En un contexto mediatizado y articulado al bagaje técnico del que se apropian las usuarias, las redes sociodigitales se plantean como este espacio visible para moldear a la esfera pública desde el discurso individual (Raimondo et. al, 2016) y desde la experiencia, donde se "involucran competencias técnicas, argumentales y políticas, y que a su vez se combinan con una creciente catarata confesional y autorreferencial de publicaciones que remiten a la mera cotidianidad" (Vázquez, 2018, p.53). Aunque algunas acotaciones que deben tomarse en cuenta son que, no toda publicación en redes sociodigitales implica participación política (Papacharissi, 2002), trasciende a la esfera pública (Raimondo et. al, 2016) o produce interés público (Vázquez, 2018).

En este análisis se identifican dos claves con las cuales se puede argumentar esta trascendencia a la esfera pública: 1) cuando las opiniones individuales se materializan en lo público, es decir, son significadas desde el interés común y se vinculan desde el desacuerdo (Vázquez, 2018), y 2) cuando adquieren visibilidad de co-presencia o visibilidad mediática (Raimondo et. al, 2016). En este ejercicio, eso conlleva a una emergencia de experiencias articuladas, lo que propone esferas públicas múltiples y alternativas, contrario a lo que originalmente suponía el modelo habermasiano de una única esfera pública (Dahlgren en Raimondo et. al, 2016), y el cual es un factor que precede a la era digital.

Una lectura crítica que es importante retomar es la propuesta por Nancy Fraser, quien ofrece historizar la esfera pública como una construcción discursiva, cuya lógica está determinada por constructos genéricos masculinos que funcionan como "mecanismos institucionales para racionalizar la dominación" (Fraser, 1990, p.59). A través de este análisis, Fraser denuncia que históricamente la esfera pública ha sido construida sin las mujeres a partir de "normas de género que exacerbaban la domesticidad femenina y una abrupta separación de la esfera pública y la privada" (Fraser, 1990, p.60).

Si bien, las mujeres han sido dejadas fuera de la esfera pública, también se han caracterizado por elaborar "estilos alternativos de comportamiento político y normas alternativas de discurso público"6 $^{\prime \prime}$ (Fraser, 1990, p.61). Como lo señala Fraser a partir de la configuración de discursos paralelos, lo que denomina contradiscursos conformados por contrapúblicos subalternos. Un ejemplo clave es el contrapúblico feminista del siglo $X X$, que han configurado lademás de una variada diversidad de productos y espacios de discusión):

en esta esfera pública, nuevos términos para describir la realidad social, incluyendo "sexismo”, “doble jornada”, “acoso sexual”, "violación matrimonial, de cita y de conocido". Armadas con este lenguaje, hemos reestructurado nuestras necesidades e identidades, reduciendo, aunque no eliminando, nuestra desventaja en las esferas públicas oficiales (p.67).

En este debate abierto, hay varias claves para comprender y analizar internet y las redes sociodigitales. La primera implica atender la diversidad que supone el análisis de sus contenidos, sus lectores/as y sus productores/as, donde no hay que dejar de lado un enfoque crítico sobre la forma en la que 
se construyen las discusiones online lquiénes participan en ellas, quiénes no, qué se dice y qué se ocultal entretejidas ante la ausencia de neutralidad. Entenderlas como espacio público, en tanto conforman puntos de organización de la experiencia colectiva (Raimondo et. al, 2016) y como parte del complejo mosaico de esferas públicas, que propone Keane "que se traslapan e interconectan" (1997, p.57). Por lo tanto, tienen cualidades potenciales para alimentar discusiones públicas que ya están siendo debatidas offline. Y son un territorio para la organización colectiva y discursiva de las mujeres desde el feminismo, donde las actoras clave son las mujeres auto-organizadas que hacen uso de sus experiencias como acto político que se propone develar que la violencia que sufren es estructural y su punto de reunión está en la dimensión online para redimensionar, nuevamente, cómo lo personal es político.

\section{Metodología}

La red se estructuró a partir del contexto en el que surgió \#MeTooEscritoresMexicanos (México) y una diversidad de hashtags derivados que llevaron a identificar patrones de organización que se articulaban discursivamente a otros hashtags nacionales e internacionales, y cuyo eje de conversación es la narración de experiencias de violencia. De esta manera, la red se construyó por medio de diversos métodos y técnicas. Uno de ellos fue el método que propone la etnografía digital, el lurking research y el ARS. De acuerdo con Christine Hine (2015) y Ángela Cora García et al (2009), la etnografía digital implica resignificar desde la etnografía la dimensión online como una realidad que también está construida a partir del lenguaje y de las vivencias de las usuarias/os, replantear el campo desde la participación del etnógrafo/a mediada por formas tecnológicas, quien participa en la reconstrucción de momentos y espacios que las usuarias/os crearon con el propósito de identificar y construir el objeto de investigación a partir de la observación y búsqueda (García et al, 2009; Hine, 2015).

De acuerdo con García, es a partir del lurking research que quien observa puede estar ahí sin participar, aunque esto conlleva también definir prácticas éticas para observar (García et al, 2009, pp.58-59) y, por lo tanto, implica una búsqueda y una mirada que no es casual, pues ya existe una noción definida de lo que se va a buscar. Posteriormente se evalúa, clasifica y sistematiza información (Strickland \& Schlesinger, 1969; García, 2009; Edelmann, 2016).

El ARS, de acuerdo con Faust (2002), es una perspectiva de análisis que considera a los individuos como actores interdependientes. Es por eso que el ARS parte de entender y establecer lazos entre entidades sociales (actores-nodos), lo que Faust denomina como conceptualización relacional de la situación. Es decir, la imagen de red obtenida debe ser entendida como un modelo, que permite establecer representaciones visuales y comprender propiedades de las relaciones entre actores para observarlas e interpretarlas (2002).

De esta manera, primero se realizó una recopilación base de hashtags, que consideró lo siguiente: (1) El hashtag surgió a partir de un conflicto social de violencia o desigualdad contra mujeres y niñas; (2) fue tendencia nacional (México), y en algunos casos, internacional; (3) los medios digitales retomaron constantemente el hashtag para hablar del tema; (4) se realizaron movilizaciones en las calles a partir del surgimiento del hashtag o funcionó para congregar a personas a la movilización; (5) el rastreo temporal ayudó a definir el 2009 como el año de inicio del hashtag \#NiUnaMás y se marcó el final de la recopilación en abril del 2019 con la ola de hashtags que surgió después de \#MeTooEscritoresMexicanos.

El resto de los hashtags fue identificado por medio de búsquedas en Twitter ${ }^{7}$ y Google que fueron direccionando a otras discusiones a nivel internacional y cuyo contexto de surgimiento fue definido por la localidad. De esta manera se determinó un patrón constante mientras se realizaba el lurking research, y fue la presencia de dos o más hashtags que acompañaban a la búsqueda de la primera ronda de hashtags.

La técnica de análisis de co-palabras "se trata de una técnica de análisis de contenido que usa patrones de co-ocurrencia de pares de ítems Ipalabras o términos) dentro de un corpus textual, para identificar las relaciones entre las ideas en un dominio determinado" (Miguel, Caprile \& Vidal, 2008), y fue la que dio la pauta para organizar y determinar relaciones direccionadas de los 47 hashtags de la muestra final. De esta forma, se sistematizó y se programó la red por medio del software Pajek (Ruiz 
El total de hashtags considerados es el siguiente:

Tabla 1: Los 47 hashtags

\begin{tabular}{|l|l|l|l|}
\hline 1 & \#NiUnaMas & 25 & \#8M \\
\hline 2 & \#25N & 26 & \#Gleichistmehr \\
\hline 3 & \#PrimeiroAssedio & 27 & \#3siSTOP \\
\hline 4 & \#NiUnaMenos & 28 & \#EqualPay \\
\hline 5 & \#BringBackOurGirls & 29 & \#WakingTheFeminist \\
\hline 6 & \#ViajoSola & 30 & \#SoyFeminista \\
\hline 7 & \#NotOkay & 31 & \#YoSiTeCreo \\
\hline 8 & \#VivasNosQueremos & 32 & \#BelieveSurvivors \\
\hline 9 & \#24A & 33 & \#MyLifelsNotYourPorn \\
\hline 10 & \#MiPrimerAcoso & 34 & \#MeTooEscritoresMexicanos \\
\hline 11 & \#BlackMonday & 35 & \#MeTooCultura \\
\hline 12 & \#WomensMarch & 36 & \#MeTooAgencias \\
\hline 13 & \#NoEsDeHombres & 37 & \#MeTooArtes \\
\hline 14 & \#SiMeMatan & 38 & \#MeTooDanza \\
\hline 15 & \#MeToo & 39 & \#MeTooMusica \\
\hline 16 & \#YoTambien & 40 & \#MeTooTeatro \\
\hline 17 & \#YaEstuvo & 41 & \#MeTooUNAM \\
\hline 18 & \#TimesUp & 42 & \#MeToolTAM \\
\hline 19 & \#Cuentalo & 43 & \#MeTooPeriodistasMexicanos \\
\hline 20 & \#TodasSomosManada & 44 & \#MeTooCreativosMexicanos \\
\hline 21 & \#HomeToVote & 45 & \#MeTooAcademicos \\
\hline 22 & \#Panuelazolnternacional & 46 & \#MeTooArtesMX \\
\hline 23 & \#SeraLey & 47 & \#MeTooActivistasMexicanos \\
\hline 24 & \#FreeTheCorset & \multirow{2}{*}{ Fuente: Tabla de elaboración propia } \\
\hline
\end{tabular}

\& Jung, 2013) y su visualización con VosViewer (Van Eck \& Waltman, 2017), mientras que la estructura fue determinada con el algoritmo Kamada Kawai por componentes separados. Kamada Kawai,

usa una analogía física de un sistema de energías potenciales para determinar la estructura de la red [...] se parte de ver a una red $G=(V, X)$ como un sistema de cuerpos con fuerzas que actúan entre ellos y lo que se busca es una configuración para la posición de cada cuerpo tal que la suma de las fuerzas que actúan sobre cada cuerpo sea cero.

\section{Análisis: La red 47 Hashtags}

La estructura de la red dio la oportunidad de identificar que las protestas feministas, por medio de la plataforma Twitter, están vinculadas entre ellas. Esta característica específica permite hablar de una estructura cognitiva cuya similaridad semántica se establece "en función del número de propiedades que los conceptos tienen en común. Mientras más propiedades tengan en común, más enlazados están mediante esas propiedades, de modo que están más próximamente relacionados" (Casas \& Luengo, 2001, p.14). 
Esto significa que, al momento de hacer una denuncia por alguna forma de violencia, las usuarias hacen referencia a otros hashtags con algún tipo de similaridad semántica. Por ejemplo, al momento en el que se impulsó \#SiMeMatan (Animal Político, 2017), que surge a raíz del feminicidio de Lesvy Berlín Osorio en Ciudad Universitaria el 3 de mayo de 2017, se hace uso de hashtags cuyo contenido sea semánticamente próximo para afirmar la denuncia. Durante el año en el que surge \#SiMeMatan, ya existía \#VivasNosQueremos, \#NiUnaMás y \#NiUnaMenos, protestas que se han hecho de uso recurrente para denunciar la violencia contras niñas y mujeres, en específico el de la violencia feminicida.
Continuando con el mismo ejemplo, \#SiMeMatan vuelve a tomar relevancia a partir del feminicidio de Mara Fernanda Castilla (Milenio, 2017) el 8 de septiembre de 2017 a bordo de un Cabify en Puebla, México. El uso del hashtag se resignifica en nuevo contexto, pero mantiene la raíz de la denuncia. Al mismo tiempo recurre a afirmarse semánticamente de hashtags como \#VivasNosQueremos, \#NiUnaMás, \#NiUnaMenos y, a partir del contexto en el que sucede, también se apela a \#ViajoSola. La estructura tiene la presencia de tres grupos temáticos que denuncian: (a) Violencia sobre los derechos reproductivos de las mujeres; (b) Violencia sobre los derechos laborales de las mujeres; (c) violencia sexual y feminicida (Ver Fig. 1).

Fig. 1 Red total. Programación con Pajek, visualización con VosViewer

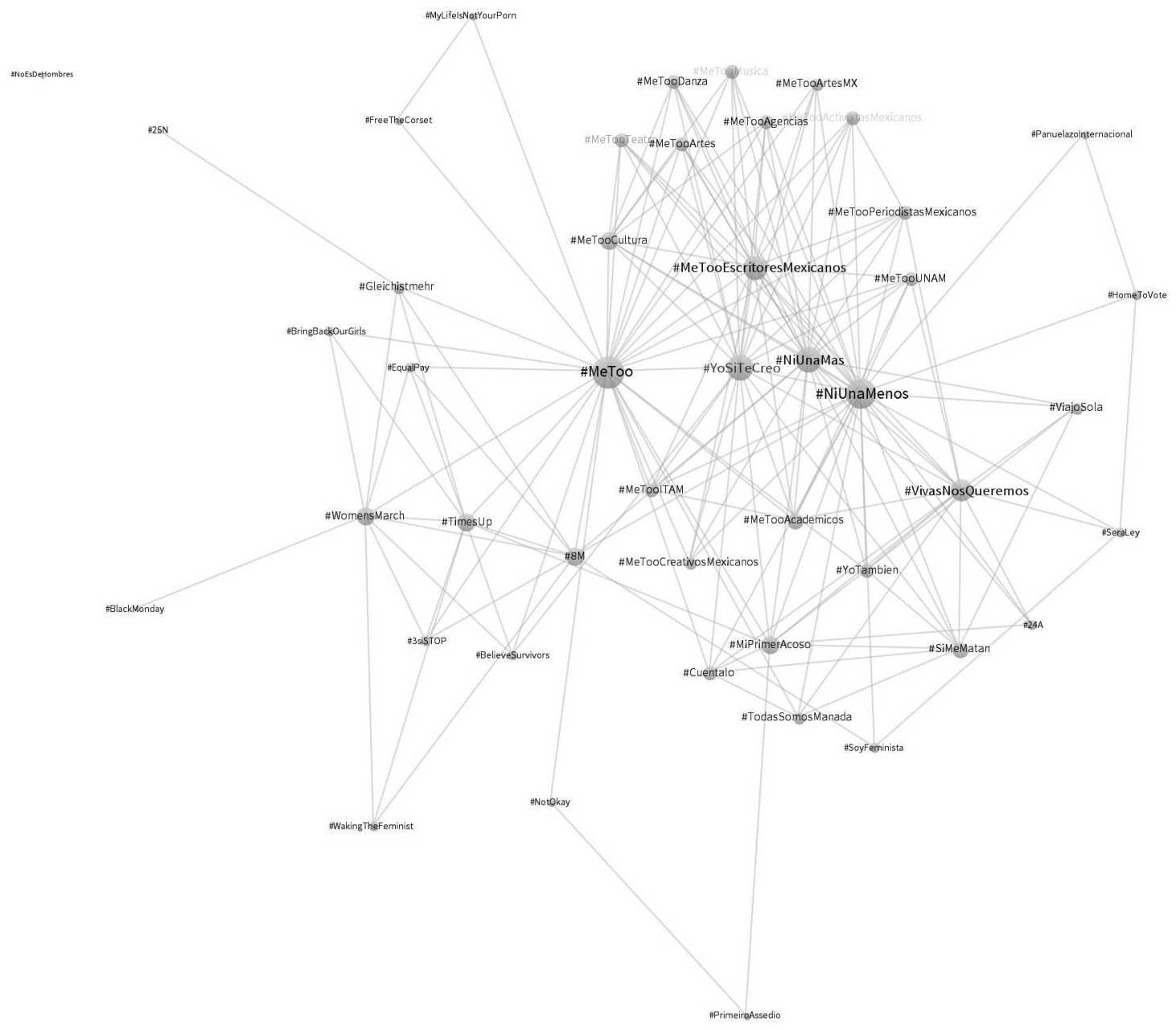




\subsection{Violencia sobre los derechos reproductivos de las mujeres}

En el lateral derecho de la red, se identifican los hashtags: \#SeráLey (Argentina), \#HomeToVote (Irlanda) y \#Pañuelazolnternacional (Argentina). Estos tres hashtags responden a un grupo temático de violencia sobre los derechos reproductivos de las mujeres durante el contexto de despenalización del aborto en Argentina (Politi \& Lodoño, 2018) e Irlanda (Mezzofiore, 2018) durante el 2018. En conjunto se vinculan semánticamente a \#NiUnaMenos $^{9}$, a partir de denunciar que, ante la negación de abortos legales y seguros, la prevalencia de la clandestinidad resulta en la muerte de niñas y mujeres.

Cabe mencionar que \#BlackMonday (Polonia) también es un hashtag que fue tendencia para denunciar los intentos de penalizar el aborto en Polonia (BBC, 2016) en octubre de 2016, pero este se localiza alejado de este grupo, en gran medida hacia la periferia de la red y con un único vínculo con \#WomensMarch. Esto se puede explicar a partir de entender el contexto en el cual surge. \#BlackMonday responde a los sucesos que ocurrían en el 2016 a nivel internacional, específicamente a las controversias surgidas durante la candidatura de Donald Trump a principios de octubre (The New York Times, 2016). A pesar de que \#WomensMarch fue organizado en enero de 2017 como protesta ante la elección de Trump como presidente de Estados Unidos y su definida agenda contra las políticas de salud sexual, las conversaciones de las usuarias en Twitter aludían a la marcha realizada en Polonia meses antes.

\subsection{Violencia sobre los derechos laborales de las mujeres}

Hay que tener ciertos parámetros al momento de definir este grupo de hashtags que se ubican en la parte izquierda inferior de la red, ya que, a pesar que se observan protestas para denunciar la violencia sobre los derechos laborales de las mujeres: \#Gleichistmehr (Röhlig, 2016) en Alemania, \#EqualPay en Estados Unidos y \#3Stop (Women-

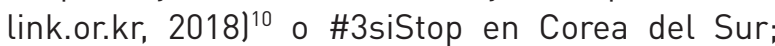
también se encuentran hashtags referentes a movimientos internacionales lque no son referentes a las denuncias por derechos laborales) y que puede ser explicado a partir del contexto en el que se viralizan en Twitter.

Por ello, en el conjunto aparecen \#BringBackOurGirls (Estados Unidos), \#BelieveTheSurvivors (Estados Unidos), \#BlackMonday (Polonia) y \#WakingTheFeminist (Irlanda). A partir de esto, es necesario establecer el sentido del análisis en comprender \#WomensMarch (Estados Unidos), \#TimesUp (Estados Unidos) y \#8M (Internacional).

Se nombró a este grupo como hashtags que denuncian la violencia sobre los derechos laborales, debido a que la principal protesta que se establece en \#WomensMarch, \#TimesUp y \#8M (Internacional), implica visibilizar la ausencia de equidad laboral. Mientras que \#WomensMarch se origina como protesta contra el gobierno de Donald Trump ${ }^{11}$ posteriormente se establece como un movimiento dinámico y plural en torno a los derechos básicos de las mujeres, entre ellos la equidad laboral. \#TimesUp ${ }^{12}$ se origina como una denuncia pública de mujeres del medio artístico hollywoodense durante los premios Oscar en enero de 2018, en torno a la ausencia de equidad salarial, al hostigamiento y violencia sexual de su ambiente laboral. El principal detonante de \#TimesUp en este contexto es \#MeToo y las acusaciones de agresión sexual contra Harvey Weinstein (Johnson \& Hawbaker, 2019).

Y finalmente está \#8M, que alude al 8 de marzo, Día Internacional de la Mujer. Este funciona como vínculo temático que se enfoca en visibilizar, en diferentes contextos, las condiciones de las mujeres en tanto salud, derechos civiles y violencia. Pero tomando en cuenta los hashtags que se vinculan, la discusión que prevalece en \#8M se refiere a los derechos laborales de las mujeres a nivel internacional. De acuerdo con la estructura de la red, las usuarias encuentran una proximidad semántica para hablar de los derechos laborales en estos tres hashtags, pero es necesario considerar también la importancia del lenguaje para contextualizar la razón del vínculo.

\subsection{Violencia sexual y feminicida}

Al centro de la red se identifican los hashtags cuyo contenido temático principal gira en torno a la vio- 
lencia sexual. Este es el caso de los hashtags cuyos nodos son visiblemente más grandes, entre ellos se encuentra \#MeToo, \#NiUnaMenos (Argentina), \#NiUnaMás (México), \#YoSíTeCreo (Internacional), \#MeTooEscritoresMexicanos (México) (Ávila, 2019) y \#VivasNosQueremos (México). A pesar de que cada uno de los hashtags surge en contextos y localidades diferentes, hay que considerar que, el síntoma de violencia sexual y feminicida se repite en América Latina donde al menos 2,795 mujeres fueron asesinadas en 2017 por razones de género en 23 países de América Latina y el Caribe. Asimismo, hay que considerar la importancia del lenguaje como un eje de apropiación en diversos países de habla hispana.

El caso de \#MeToo es particular, ya que es un nodo que vincula la discusión internacional en torno a la violencia sexual con la discusión hispanohablante, en tanto se relaciona con \#YoSíTeCreo y la ola de hashtags sobre \#MeToo en México (Ávila, 2019) a partir de la apropiación y resignificación que le dieron las usuarias de acuerdo con diferentes espacios laborales.

Es, por tanto, importante reconsiderar el papel que tiene un hashtag como lo es \#YoSíTeCreo en la protesta contra la violencia sexual. En este contexto, el ejercicio que realizan las usuarias al vincular semánticamente \#YoSíTeCreo, implica una estrategia de apoyo ante la revictimización que experimentan las mujeres que narran sus vivencias de acoso, hostigamiento y agresión sexual en el caso específico de Twitter. Esta forma de apoyo parte de un ejercicio ético y político de sororidad, a través de la "solidaridad entre mujeres [...] un camino real para ocupar espacios, lograr derechos, consolidar protecciones entre mujeres y eliminar el aislamiento, la desvalía y el abandono" (Lagarde, 2012, p. 34).

La red de la figura 1 se aplicó en VosViewer clustering y el resultado es la figura 2. Este algoritmo fue aplicado porque permite "visualizar los grupos de nodos con mayor cercanía entre ellos y se determina por parámetros de resolución, mientras mayor sea este parámetro, mayor será el número de grupos" (Van Eck \& Waltman, 2014, p.10). Asimismo, se ajustó la escala de visualización y el tamaño de variación de las etiquetas. Ambas herramientas ayudan a definir la visualización de acuerdo con la importancia que tienen los nodos dentro de la totalidad de la red.

Como es posible observar, la aplicación del clustering corroboró, por una parte, estos tres grupos

Fig. 2 Clusters. Programación con Pajek

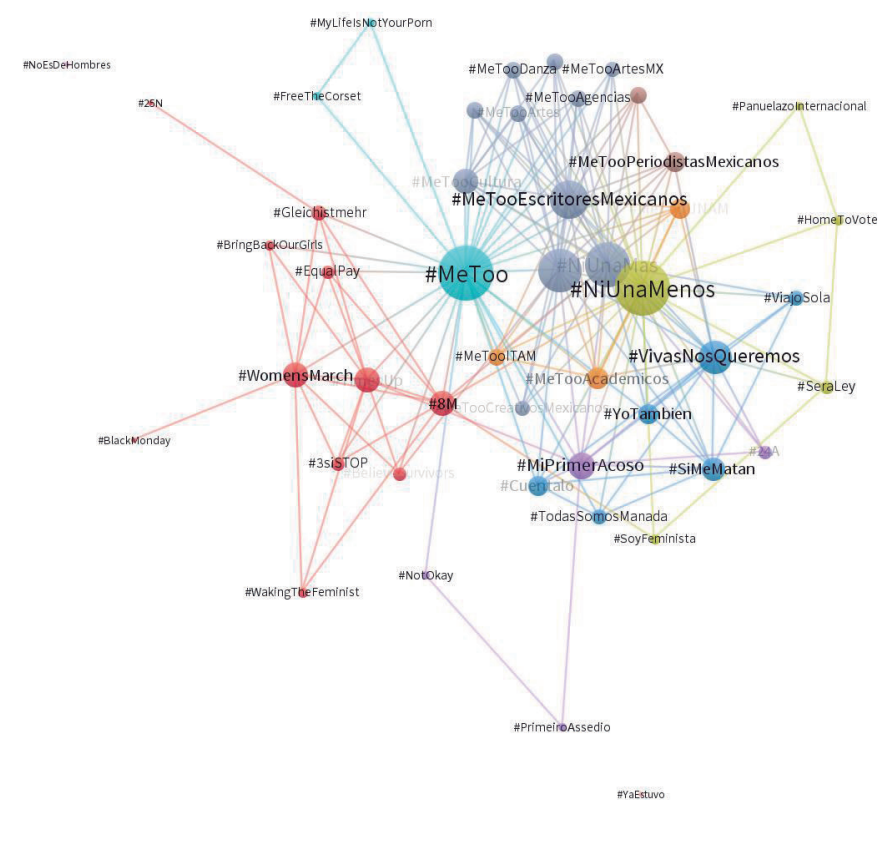


temáticos, pero al mismo tiempo se hacen notar subgrupos, especialmente se distinguen len Méxicol: \#MeTooUNAM, \#MeToolTAM, \#MeTooAcadémicos en naranja; en la parte superior en índigo se ubican: \#MeTooCultura, \#MeTooArtes, \#MeTooAgencias, \#MeTooArtesMx, \#MeTooMúsica, \#MeTooTeatro; $y$, finalmente, el subgrupo en azul que condensa \#VivasNosQueremos, compuesto por \#Viajo Sola (Argentina - Internacional), \#YoTambién (Internacional), \#SiMeMatan (México), \#Cuéntalo (España) y \#TodasSomosManada (España).

En el análisis se observa cómo los subgrupos -específicamente en los derivados de \#MeToo- están determinados por los espacios a los que se refieren y por el ejercicio de sororidad por parte de las usuarias para la visibilización de cómo la violencia se reproduce en espacios académicos y culturales. Esto trae a debate: 1) denunciar a los agresores que repiten y ejercen prácticas de acoso y hostigamiento, y 2) que los agresores están en posibilidad de repetir estás prácticas en gran variedad de instituciones, gracias a las estructuras de poder que los respaldan a pesar de la existencia de denuncias que preceden a estos hashtags.

Finalmente, el subgrupo azul está representado por el nodo principal de \#VivasNosQueremos. Como se mencionó anteriormente, estas relaciones semánticas se definen por el contexto en el que surgen y el uso que le dan las usuarias, en tanto se apoyan en hacer referencia a una protesta política común. Este es el caso de \#VivasNosQueremos (Torres, 2018), cuyo origen viene de la ola de feminicidios en Ciudad Juárez desde 1993. Lo anterior da una pista para repensar cómo se están adaptando las protestas de origen feminista a espacios públicos como lo son internet y redes sociodigitales.

\section{Conclusiones}

En esta red de 47 hashtags es posible interpretar que las usuarias de Twitter continúan, no sólo generando nuevas formas de denunciar diferentes tipos de violencia, sino que hacen uso de hashtags que han marcado paradigmas discursivos y definen la agenda feminista en redes sociodigitales. En tanto congregan lingüística y contextualmente un vehículo con sentido político definido en el espacio público de internet.

En el caso de \#MeToo, una de las características principales ha sido utilizar la propia experiencia como herramienta de denuncia pública, determinada por un contexto jurídico, legal, social, económico y cultural que no protege, ni les cree a las mujeres cuando denuncian la violencia sexual que vivieron. El idioma también representa un punto de partida que ayuda a vincular experiencias internacionales y que, por lo tanto, se resignifican de acuerdo con las necesidades de las propias localidades. Este es el caso de \#MeTooEscritoresMexicanos (México) y el resto de hashtags que particularizaron las violencias sexuales en diversos ámbitos laborales. Asimismo, es el caso de \#FreeTheCorset y \#MyLifelsNotYourPorn (Corea del Sur) que generó una serie de movimientos, especialmente en Seúl, para oponerse a los estereotipos de belleza de las mujeres y la invasión de su privacidad.

Por otra parte, \#NiUnaMenos (Argentina), que se entiende a partir de su origen en \#NiUnaMás (Méxicol, y a partir del aumento de violencia feminicida en América Latina, establece un sentido positivo sobre el deseo de que las mujeres ya no sean restadas en vida, ni sumadas en cifras. Pero la razón del constante vínculo de este hashtag (\#NiUnaMenos) con otros en la red, ayuda a contextualizar, no sólo el feminicidio, también lo hace con las muertes derivadas de la ausencia de derechos reproductivos, la falta de acceso a un sistema de salud que no denigre ni violente a las mujeres, así como el acceso a derechos laborales, a la participación civil y política, etc.

Con este panorama, se puede proponer que los movimientos feministas en internet y redes sociodigitales tienen una estructura cognitiva que no es casual ni espontánea, más bien se encuentra en un constante proceso de organización colectiva y discursiva en la esfera pública. Un movimiento digital, social y global que produce nuevos imaginarios desde el razonamiento feminista para nombrar lo que es invisible a través de la autovinculación y la autoorganización ${ }^{13}$. 


\section{Notas}

1 La traducción es mía.

2 Aunque en esta revisión se hace un posicionamiento positivo acerca del rol de internet y redes sociodigitales, no se deja de lado elementos críticos hacia las condiciones comerciales, políticas y de vigilancia de la web.

3 La traducción es mía.

4 Tanto Castaño y Vázquez (2011) como Torregrosa (2011) definen que hay diversos niveles de brechas digitales. La que está limitada por el acceso a infraestructura y la del uso. A la brecha digital de uso, Castaño y Vázquez la denominan 'brecha de género'.

5 Es necesario agregar que en ningún momento mi intención es dejar de lado las brechas de acceso y los sesgos de uso de internet y redes sociodigitales. El análisis de acceso es prioritario cuando se estudian dinámicas en la dimensión online, lo que nos permite no sólo elaborar estudios éticos y políticos sobre quiénes son los que tiene control y conocimientos de estos espacios, sino que también posibilita vincular nuestros análisis con lo que sucede en la dimensión offline.

6 La traducción es mía.

7 Los argumentos sobre los cuales decidí usar Twitter se encuentran en que el uso del hashtag (\#) es una propuesta originaria de esta plataforma. Asimismo, el rastreo en términos cualitativos la través de la herramienta de búsqueda avanzada de la misma plataformal ha resultado más eficiente a partir del método del lurking research propuesto desde la etnografía digital. Ha sido de bajo costo y ha permitido vincular los hashtags por medio de la metodología propuesta de análisis de co-palabras.

8 Para la programación de la red no se usaron acentos y los hashtags en hangul fueron romanizados.

9 Ni una menos, manifiesto (2015). URL: http:// niunamenos.org.ar/manifiestos/3-de-junio-2015/

10 \#3Stop, fue una movilización de mujeres coreanas en marzo de 2018. Detuvieron todas sus actividades a las 3 de la tarde para protestar contra la brecha salarial, la doble jornada laboral que implica el cuidado doméstico y el hostigamiento laboral en Corea del Sur. El detonante fue \#MeToo. [3STOP]! (2018). URL: http:// www.womenlink.or.kr/statements/19912

11 History. This day in history: Women's March (2017). Dirección URL: https://www.history.com/this-day-inhistory/womens-march

12 Time's Up (2019). Dirección URL: https://www. timesupnow.com

13 Es importante notar que este artículo propone analizar cómo esta serie de hashtags sirven para organizar discursivamente lo que está invisibilizado por las estructuras definidas de poder patriarcal. No se busca dejar de lado que hay una reapropiación y resignificación por parte de otros diversos grupos con fines completamente diferentes a la reivindicación de las mujeres. Estos tienen que contextualizarse de acuerdo con los objetivos que se quieran analizar, pero principalmente es importante historizar cómo y quiénes han definido su impacto social.

\section{Referencias}

Animal Político (2017). \#SiMeMatan, la reacción de mujeres ante la criminalización de una joven que llevó a la PGJ a rectificar. En Animal Político En línea. URL: https:// www.animalpolitico.com/2017/05/simematan-feminicidio-mexico-unam/

Ávila, Y. (2019) ¿Cómo surgió el movimiento \#MeToo y cómo revivió en México? En Animal Político. URL: https://www.animalpolitico.com/elsabueso/como-surgio-el-movimiento-me-too-y-como-revivio-en-mexico/

BBC (2016). Black Monday: Polish women strike against abortion ban. En BBC News. URL: https://www.bbc.com/news/world-europe-37540139

Casas, L. \& Luengo, R. (2001). Aportaciones a la investigación sobre la estructura cognitiva de los alumnos a través de redes de Pathfinder. Un estudio exploratorio en Geometría. Programa de doctorado. Mayo. Badajoz. URL: https://www.uv.es/aprengeom/archivos2/Casas01.pdf

Castañeda, M. (2008). Metodología de la investigación feminista. México: CEIICH-UNAM. 
Castaño, C. \& Vázquez, S. (2011). La brecha digital de género: prácticas de e-inclusión y razones de la exclusión de las mujeres. Asparkía, Revista de investigación feminista, 22, 33-49.

Castells, M. (2009). Comunicación y Poder. Madrid: Alianza Editorial.

Castells, M. (2010). The rise of the network society. Reino Unido: Blackwell Publishing Ltd.

CEPAL (2018). Feminicidio. URL: https://oig.cepal.org/es/indicadores/feminicidio CNN (2011). Egypt's million woman march fizzles into shouting matches. En línea. URL: http://edition.cnn.com/2011/WORLD/meast/03/08/egypt.women/index. html

Cobo, R. (2008). Patriarcado y feminismo: del dominio a la rebelión. El valor de la palabra. Revista anual de pensamiento. Hacia la ciudadanía del siglo XXI, 6.

De las Heras, S. (2008). Una aproximación a las teorías feministas. Universitas. Revista de Filosofía, Derecho y política, 9, 45-82. http://universitas.idhbc.es/n09/09-05.pdf

De Miguel, A. \& Boix, M. (2002). Los géneros de la red: los ciberfeminismos. Mujeres en red. En línea. URL: http://www.mujeresenred.net/spip.php?article297

De Miguel, A. (2011) Mujeres en Red. Los feminismos a través de la historia. En línea. URL: http://www.mujeresenred.net/anademiguel.html

De Miguel, A. (2008). La violencia contra las mujeres. Tres momentos en la construcción del marco feminista de interpretación. ISEGORÍA, 38, enero-junio, 2008, 129137, ISSN: 1130-2097

Edelmann, N. (2016). What is Lurking? A Literature Review of Research on Lurking. En Riva, G.; Wirderhold, B. y Cipresso, P. (2016). The Psychology of Social Networking, (1). DOI https://doi.org/10.1515/9783110473780-015

Faust, K. (2002). "Las redes sociales en las ciencias sociales y del comportamiento". En Gil, J. \& Schmidt, S. (eds.), Análisis de Redes Sociales: Aplicaciones en Ciencias Sociales, IIMAS, UNAM, México, 1-14.

Fernández, V. (2012). Un acercamiento a la experiencia en clave feminista. Práctica y usos estratégicos de la red. Temas de Comunicación, 24, 62-78.

Fraser, N. (1990). Rethinking the Public Sphere: A Contribution to the Critique of Actually Existing Democracy. Social Text, 25/26, 56-80.

García, A.; Standlee, A.; Bechkoff, J. y Cui, Y. (2009). Ethnographic Approaches to the Internet and Computer-Mediated Communication. Journal of Contemporary Ethnography, 38 (1), 52. DOI: 10.1177/0891241607310839

Habermas, J.; Lennox, S. y Lennox, F. (1964). The Public Sphere: An Encyclopedia Article. New German Critique, 3, 49-55. En línea. URL: http://www.jstor.org/stable/487737

Haraway, D. (1991). Ciencia, ciborgs y mujeres. La reinvención de la naturaleza. Madrid: Ediciones Cátedra.

Harding, S. (1998). ¿Existe un método feminista? En Bartra, E. (1998). Debates en torno a una metodología feminista. Ciudad de México: PUEG-UAM.

Hine, C. (2015). Ethnographic for the Internet. Embedded, Embodied and Everyday. Londres: 
Bloomsbury Academic.

Johnson, C. \& Hawbaker, K. (2019). \#MeToo: A timeline of events. En Chicago Tribune. URL: https://www.chicagotribune.com/lifestyles/ct-me-too-timeline-20171208-htmlstory.html

Keane, J. (1997). Transformaciones estructurales de la esfera pública. Estudios Sociológicos, 43, 47-77.

Khan, M. Z.; Gilani, I. S, y Nawaz, A. (2012). From Habermas Model to New Public Sphere: A Paradigm Shift. Global Journal of Human Social Science. (12) 5.

Kranzberg, M. (1985). The information age: Evolution or revolution? En Guile, B. (Ed.) Information technologies and social transformation. Washington, DC: National Academy Press.

Lagarde, M. (1996). Género y feminismo. Madrid: Ed. Horas y Horas.

Lagarde, M. (2012). El feminismo en mi vida. Hitos, claves y topias. Ciudad de México: INMUJERES- Gobierno de la Ciudad de México.

Marin, A. \& Wellman, B. (2011). “Social Network Analysis: An introduction.” En Scott, J. \& Carrington, P. Sage Handbook of Social Network Analysis. Londres: Sage Publications Ltd.

Mezzofiore, G. (2018). Irish across world travel \#HomeToVote in landmark abortion referendum. En CNN World. URL: https://edition.cnn.com/2018/05/24/europe/ hometovote-ireland-abortion-referendum-intl/index.html

Miguel, S., Caprile, L. \& Vidal, I. (2008). Análisis de co-términos y de redes sociales para la generación de mapas temáticos. El profesional de la información, 17 (6), 637646. En Memoria Académica. En línea: http://www.memoria.fahce.unlp.edu.ar/ art_revistas/pr.6387/pr.6387.pdf

Milenio (2017). \#SiMeMatan, el hashtag que deberíamos dejar de usar. En Milenio Digital. En línea. URL: http://www.milenio.com/estilo/simematan-el-hashtag-quedeberiamos-dejar-de-usar

Mohanty, C. (2003). Feminism without borders. Decolonizing theory, practicing solidarity. Durham \& London: Duke University.

Papacharissi, Z. (2002). The virtual sphere: the internet as a public sphere. New Media and Society. 4 (1), 9-27. DOI: 10.1177/14614440222226244

Papacharissi, Z. (2009). The Virtual Sphere 2.0: The Internet, the Public Sphere and beyond. En Chadwick, A. \& Howard, P. Londres: Routledge Handbook Internet Politics.

Papacharissi, Z. (2011). On convergent supersurfaces and public spheres online. International Journal of Electronic Governance, (4) 1/2, 9-17. DOI: 10.1504/IJEG.2011.041704

Politi, D. \& Lodoño, E. (2018). El aborto no fue legalizado en Argentina, pero la lucha que impulsó no se detiene. En New York Times. URL: https://www.nytimes.com/ es/2018/08/10/aborto-argentina-senado/

Raimondo A., N.; Reviglio, M. y Divani, R. (2016). Esfera pública y redes sociales en Internet: ¿Qué es lo nuevo en Facebook? Revista Mediterránea de Comunicación, 7(1), 211-229. DOI: http://dx.doi.org/10.14198/MEDCOM2016.7.1.12 
Röhlig, M. (2016). Frauen werden in Deutschland viel schlechter bezahlt als Männer. En Bento. URL: https://www.bento.de/politik/equal-payfrauen-verdienen-in-deutschland-21-prozent-weniger-als-maenner -a-00000000-0003-0001-0000-000000655957

Ruiz, A. \& Jung, N. (2013) Visualización con Pajek. SUNBELT XXXIII. Laboratorio de redes IIMAS-UNAM.URL: http://mrvar.fdv.uni-lj.si/pajek/spanish/Spanish.pdf

Ruiz, A. \& Russell, J. (2016). La estructura del sistema científico de México a finales del siglo XX: una visión a nivel de instituciones. Redes. Revista Hispana para el Análisis de Redes Sociales, 27 (2), p. $11-32$.

Scott, J. (1997) El género: una categoría útil para el análisis histórico. En Lamas, M. (comp.) El género, La construcción cultura de la diferencia. Ciudad de México: UNAM-PUEG, pp. 265-302.

Solares, B. (1997). El síndrome Habermas. Ciudad de México: Facultad de Ciencias Políticas y Sociales. UNAM.

Stone-Mediatore, S. (1999). Chandra Mohanty y la revalorización de la "experiencia". En Hiparquia, vol. X, julio. URL: http://www.hiparquia.fahce.unlp.edu.ar/numeros/ volx/chandra-mohanty-y-la-revalorizacion-de-la-experiencia\#sdfootnote1sym

Strickland, D. \& Schlesinger, L. (1969). “Lurking” as a Research Method. Human Organization: Fall 1969, (28) 3, pp. 248-250.

Thompson, J. (2011). Los límites cambiantes de la vida pública y la privada. Comunicación y Sociedad, Nueva Época, 15, 11-42.

Toret, J. (2013). Tecnopolítica: la potencia de las multitudes conectadas. El sistema red 15M, un nuevo paradigma de la política distribuida. Barcelona: IN3 Working Paper Series.

Torregrosa. J. (2011). Brecha digital. Notas desde una perspectiva de género. Asparkía, 22, 51-59.

Torres, C. (2018). Susana Chávez, un origen de "ni una menos”. La Izquierda Diario. URL: https://www.laizquierdadiario.com/Susana-Chavez-un-origen-de-NiUnaMenos.

The New York Times (2016). Transcript: Donald Trump's Taped Comments About Women. En The New York Times. URL: https://www.nytimes.com/2016/10/08/us/donaldtrump-tape-transcript.html

Valcárcel, A. (2001). La memoria colectiva y los retos del feminismo. Chile: Unidad Mujer y Desarrollo, CEPAL. URL: http://kolectivoporoto.cl/wp-content/uploads/2015/11/ Valcarcel-Amelia-La-memoria-colectiva-y-los-retos-del-feminismo.pdf

Van Dijk, J. (2006) The network society. Social aspects of new media. Londres: SAGE Publications.

Van Eck, N., \& Waltman, L. (2014). Visualizing bibliometric networks. En Ding, Y., Rousseau, R. \& D. Wolfram (Eds.), Measuring scholarly impact: Methods and practice (pp. 285-320). Springer. Disponible en: http://www.vosviewer.com/download/fx2.pdf 
Van Eck, N.J., \& Waltman, L. (2017) VOSviewer Manual. Universiteit Leinen. CWTS. En línea. URL: www.vosviewer.com/download/f-03u2.pdf (Consulta mayo 2019).

Vázquez, M, (2018). Esfera pública virtual. Una lectura política y comunicacional de lo público en la web. INMediaciones de la Comunicación, (13) 1, 49-69. DOI: https:// doi.org/10.18861/ic.2018.13.1.2825

Wilding, F. (1998). Where is the feminism in Cyberfeminism? N.Paradoxa, Vol. 2. En línea. URL: https://www.ktpress.co.uk/pdf/vol2_npara_6_13_Wilding.pdf

- Sobre la autora:

Daniela Esquivel es Licenciada en Comunicación y estudiante de maestria en el Posgrado de Ciencias Políticas y Sociales, UNAM, con orientación en comunicación y género.

- ¿Como citar?

Esquivel, D. (2019). Construcción de la protesta feminista en hashtags: aproximaciones desde el análisis de redes sociales. Comunicación y Medios, (40), 184-198. 